\title{
Fixed points of symplectic periodic flows
}

\author{
Alvaro Pelayo * and Susan Tolman ${ }^{\dagger}$
}

\begin{abstract}
The study of fixed points is a classical subject in geometry and dynamics. If the circle acts in a Hamiltonian fashion on a compact symplectic manifold $M$, then it is classically known that there are at least $\frac{\operatorname{dim} M}{2}+1$ fixed points; this follows from Morse theory for the momentum map of the action. In this paper we use Atiyah-Bott-Berline-Vergne (ABBV) localization in equivariant cohomology to prove that this conclusion also holds for symplectic circle actions with non-empty fixed sets, as long as the Chern class map is somewhere injective - the Chern class map assigns to a fixed point the sum of the action weights at the point. We complement this result with less sharp lower bounds on the number of fixed points, under no assumptions; from a dynamical systems viewpoint, our results imply that there is no symplectic periodic flow with exactly one or two equilibrium points on a compact manifold of dimension at least eight.
\end{abstract}

\section{Introduction}

The study of fixed points of flows or maps is a classical and important topic studied in geometry and dynamical systems. For instance, in the context of symplectic geometry, it follows from the works of Atiyah [1], Guillemin-Sternberg [8], and Kirwan [9] that a lot of the information of a symplectic manifold equipped with a Hamiltonian torus action is encoded by the fixed point set of the action. For example, if the torus has precisely half the dimension of the manifold, then all the information is encoded by the fixed point set; see Delzant [4].

In this paper we study symplectic (but not necessarily Hamiltonian) circle actions on compact symplectic manifolds with non-empty fixed point sets. Specifically, we are interested in providing lower bounds on the number of fixed points of the circle action. Equivalently, out goal is to give lower bounds for the number of equilibrium points of symplectic periodic flows.

There are many non-Hamiltonian circle actions with no fixed points; for instance, given an even-dimensional torus, consider the natural action of any circle subgroup. A well-known theorem of McDuff [10, Prop. 2] says that a symplectic circle action on a four-dimensional compact symplectic manifold with at least one fixed point is Hamiltonian. Non-Hamiltonian symplectic circle actions on higher dimensional manifolds are not well understood. A manifestation of this fact is that it is not known whether there is a non-Hamiltonian symplectic circle action on a compact symplectic manifold $M$ so that the fixed set $M^{S^{1}}$ is discrete but not empty. (In [10, Prop. 1 and Sec. 3], McDuff also constructs a non-Hamiltonian symplectic circle action on a six-dimensional compact symplectic manifold, but the fixed sets are tori.)

It is a classical fact that a Hamiltonian circle action on a compact symplectic manifold $(M, \omega)$ has at least $\frac{1}{2} \operatorname{dim} M+1$ fixed points. This is an easy consequence of the fact that, if the fixed set is discrete, then

* partially supported by an NSF postdoctoral fellowship; this work started when he was affiliated with MIT.

${ }^{\dagger}$ partially supported by National Science Foundation Grant DMS \#07-07122. 
the momentum map $\mu: M \rightarrow \mathbb{R}$ is a perfect Morse function whose critical set is the fixed set. Therefore, the number of fixed points is equal to the rank of $\sum_{i} \mathrm{H}_{i}(M ; \mathbb{R})$. Finally, this sum is at least $\frac{1}{2} \operatorname{dim} M+1$ because

$$
[1],[\omega],\left[\omega^{2}\right], \ldots,\left[\omega^{\frac{1}{2} \operatorname{dim} M}\right]
$$

are distinct cohomology classes. In addition, a lot of properties of Hamiltonian circle actions are known, see McDuff-Tolman [11] and the references therein. A natural question arises: what can one say about the fixed point set of a symplectic circle action? The goal of this paper is to shed light into this question. Before stating our main results we need to recall some terminology.

A symplectic form on a compact manifold $M$ is a closed, non-degenerate two-form $\omega \in \Omega^{2}(M)$. A circle action on $M$ is symplectic if it preserves $\omega$. A symplectic circle action is Hamiltonian if there exists a map $\mu: M \rightarrow \mathbb{R}$ such that

$$
-\mathrm{d} \mu=\iota_{X_{M}} \omega
$$

where $X_{M}$ is the vector field on $M$ induced by the circle action. The map $\mu$ is called the momentum map. Since $\iota_{X_{M}} \omega$ is closed, every symplectic action is Hamiltonian if $\mathrm{H}^{1}(M ; \mathbb{R})=0$.

Let the circle act on a compact symplectic manifold $(M, \omega)$ with momentum map $\mu: M \rightarrow \mathbb{R}$. Since the set of compatible almost complex structures $J: \mathrm{T} M \rightarrow \mathrm{T} M$ is contractible, there is a well-defined multiset of integers, called weights, associated to each fixed point $p$. Moreover, let $\left.\mathrm{c}_{1}(M)\right|_{p}$ denote the first equivariant Chern class of the tangent bundle of $M$ at $p \in M^{S^{1}}$, which we can naturally identify with an integer $c_{1}(M)(p)$ : the sum of the weights at $p$; (see Section 2 for a brief explanation.) Under this identification, we consider the map

$$
\mathrm{c}_{1}(M): M^{S^{1}} \rightarrow \mathbb{Z}, \quad p \mapsto \mathrm{c}_{1}(M)(p) \in \mathbb{Z}
$$

We refer to the map $c_{1}(M)$ as the Chern class map of $M$. Finally, let $X$ and $Y$ be sets and let $f: X \rightarrow Y$ be a map. We recall that $f$ is somewhere injective if there is a point $y \in Y$ such that $f^{-1}(\{y\})$ is the singleton. Note that if $f$ is a somewhere injective map, then necessarily $X \neq \emptyset$. (In particular, if the Chern class map is somewhere injective, $M^{S^{1}} \neq \emptyset$.) On the other hand, if $X \neq \emptyset$, then every injective map is somewhere injective.

Theorem 1. Let the circle act symplectically on a compact symplectic manifold $M$. If the Chern class map is somewhere injective, then the circle action has at least $\frac{1}{2} \operatorname{dim} M+1$ fixed points.

There has been a considerable amount of interest, as far as we know with no success, in trying to construct non-Hamiltonian symplectic circle actions with non-empty finite fixed point set. The theorem above tells us where we should not look for examples. An interesting open question is whether Theorem 1 holds without the assumption on the Chern class map; we suspect that the answer is "yes". Our proof uses the Atiyah-Bott and Berline-Vergne localization formula in equivariant cohomology, following the ideas of the paper by Tolman-Weitsman [16] on semifree actions. We also use some basic properties of Poincaré polynomials and Morse functions to prove:

Theorem 2. If the circle acts symplectically on a compact symplectic manifold $M$ with isolated fixed points, then

$$
\sum_{p \in M^{S^{1}}} \mathrm{c}_{1}(M)(p)=0 .
$$


In their paper, Tolman and Weitsman pointed out that their results did not rule out the existence of an example of a symplectic circle action on a 6-dimensional manifold with exactly two fixed points with weights $(1,1,-2)$ and $(-1,-1,2)$ and the question has been open ever since. Note that our results above do not rule out the existence of such an example either. In this paper we shall prove:

Theorem 3. Let the circle act symplectically on a compact, connected symplectic manifold $M$ with exactly two fixed points. Then either $M$ is the 2-sphere or $\operatorname{dim} M=6$ and there exist natural numbers $a$ and $b$ so that the weights at the two fixed points are $\{a, b,-a-b\}$ and $\{a+b,-a,-b\}$.

As a conclusion from these three theorems we obtain:

Corollary 4. Let the circle act symplectically on a compact symplectic manifold $M$ with non-empty fixed point set. Then there are at least two fixed points, and if $\operatorname{dim} M \geq 8$, then there are at least three fixed points. Moreover, if the Chern class map is not identically zero and $\operatorname{dim} M \geq 6$, then there are at least four fixed points.

Proof. By Theorem 1 there cannot be exactly one fixed point; since $M^{S^{1}} \neq \emptyset$ this implies that there are least two fixed points. If $\operatorname{dim} M \geq 8$ then Theorem 3 implies that there are at least three fixed points. If the Chern class map is not identically zero, it follows from Theorem 2 that it is not constant, that is,

$$
\left|\left\{\mathrm{c}_{1}(M)(p) \mid p \in M^{S^{1}}\right\}\right| \geq 2 .
$$

Hence, if there are less than $\frac{1}{2} \operatorname{dim} M+1$ fixed points, then Theorem 1 implies that there must be at least four.

Remark 5 We do not know if there is a symplectic circle action on a compact, connected symplectic manifold $M$ with exactly three fixed points, other than the standard actions on $\mathbb{C} P^{2}$. Note that, by Corollary 12 , if this does occur then $\frac{1}{2} \operatorname{dim} M$ is even.

There are several works on (possibly non-Hamiltonian) symplectic circle actions that are related to this note, including Godinho [7] and Weitsman [18]. For works on (possibly non-Hamiltonian) symplectic $\left(S^{1}\right)^{k}$-actions, $k>1$, see Duistermaat-Pelayo [5] and Pelayo [12]; in these cases the fixed point sets are well understood. In addition, the study of $S^{1}$-actions and their flows is central in the analysis of bifurcation diagrams in the modern global theory of integrable systems, see [17], [13], [14]. A symplectic periodic flow is a flow generated by a symplectic circle action. Fixed points of the circle action correspond to equilibrium points of the flow. From a dynamical systems viewpoint, Corollary 4 implies:

Corollary 6. There is no symplectic periodic flow with exactly one equilibrium point on a compact manifold M. Moreover if $\operatorname{dim} M \geq 8$, then there is no symplectic periodic flow with exactly two equilibrium points.

Acknowledgements. Special thanks to D. McDuff for comments and giving us a stronger formulation of Theorem 1. Thanks to D. Auroux, V. Guillemin and S. Sabatini for comments on a preliminary version. Finally, we are grateful to an anonymous referee for interesting comments and suggestions. 


\section{Proofs of Theorems 1 and 2}

Before beginning these proofs, we briefly introduce some background material. Let the circle act on a manifold $M$. The equivariant cohomology of $M$ is, by definition, $\mathrm{H}_{S^{1}}^{*}(M)=\mathrm{H}^{*}\left(M \times{ }_{S^{1}} S^{\infty}\right)$. For example, if $p$ is a point then $\mathrm{H}_{S^{1}}^{*}(p ; \mathbb{Z})=\mathrm{H}^{*}\left(\mathbb{C} P^{\infty} ; \mathbb{Z}\right)=\mathbb{Z}[t]$. If $V$ is an equivariant vector bundle over $M$, then the equivariant Euler class of $V$ is the Euler class of the vector bundle $V \times{ }_{S^{1}} S^{\infty}$ over $M \times{ }_{S^{1}} S^{\infty}$. The equivariant Chern classes of equivariant complex vector bundles are defined analogously.

If $M$ is oriented and compact then the projection map $\pi: M \times{ }_{S^{1}} S^{\infty} \rightarrow \mathbb{C} P^{\infty}$ induces a natural pushforward map

$$
\pi_{*}: \mathrm{H}_{S^{1}}^{i}(M ; \mathbb{Z}) \rightarrow \mathrm{H}^{i-\operatorname{dim} M}\left(\mathbb{C} P^{\infty} ; \mathbb{Z}\right) .
$$

Note, in particular that if $i<\operatorname{dim} M$ then $\pi_{*}(\alpha)=0$ for all $\alpha \in \mathrm{H}_{S^{1}}^{i}(M ; \mathbb{Z})$. Since this map is given by "integration over the fiber," we will usually denote it by the symbol $\int_{M}$. We will need the following theorem, due to Atiyah-Bott and Berline-Vergne [2, 3].

Theorem 7 (ABBV Localization). Let the circle act on a compact oriented manifold $M$. Fix $\alpha \in \mathrm{H}_{S^{1}}^{*}(M ; \mathbb{Q})$. As elements of $\mathbb{Q}(t)$,

$$
\int_{M} \alpha=\sum_{F \subset M^{S^{1}}} \int_{F} \frac{\left.\alpha\right|_{F}}{\mathrm{e}_{S^{1}}\left(\mathrm{~N}_{F}\right)},
$$

where the sum is over all fixed components, and $\mathrm{e}_{S^{1}}\left(\mathrm{~N}_{F}\right)$ denotes the equivariant Euler class of the normal bundle to $F$.

Now let the circle act symplectically on a symplectic manifold $(M, \omega)$, and let $J: \mathrm{T} M \rightarrow \mathrm{T} M$ be a compatible almost complex structure. If $p \in M^{S^{1}}$ is an isolated fixed point, then there are well-defined nonzero (integer) weights $\xi_{1}, \ldots, \xi_{n}$ in the isotropy representation $\mathrm{T}_{p} M$ (repeated with multiplicity). Indeed, there exists an identification of $\mathrm{T}_{p} M$ with $\mathbb{C}^{n}$, where the $S^{1}$ action on $\mathbb{C}^{n}$ is given by $\lambda \cdot\left(z_{1}, \ldots, z_{n}\right)=$ $\left(\lambda^{\xi_{1}} z_{1}, \ldots, \lambda^{\xi_{n}} z_{n}\right)$; the integers $\xi_{1}, \ldots, \xi_{n}$ are determined, up to permutation, by the $S^{1}$-action and the symplectic form. Therefore, restriction of the $i^{\text {th }}$-equivariant Chern class to a fixed point $p$ is given by

$$
\left.\mathrm{c}_{i}(M)\right|_{p}=\sigma_{i}\left(\xi_{1}, \ldots, \xi_{n}\right) t^{i}
$$

where $\sigma_{i}$ is the $i$ 'th elementary symmetric polynomial and $t$ is the generator of $\mathrm{H}_{S^{1}}^{2}(p ; \mathbb{Z})$. For example, $\left.\mathrm{c}_{1}(M)\right|_{p}=\sum \xi_{i} t$ and the equivariant Euler class of the tangent bundle at $p$ is given by $\mathrm{e}_{S^{1}}\left(\mathrm{~N}_{p}\right)=$ $\left.\mathrm{c}_{n}(M)\right|_{p}=\left(\prod \xi_{j}\right) t^{n}$. Hence,

$$
\int_{p} \frac{\left.\mathrm{c}_{i}(M)\right|_{p}}{\mathrm{e}_{S^{1}}\left(\mathrm{~N}_{p}\right)}=\frac{\sigma_{i}\left(\xi_{1}, \ldots, \xi_{n}\right)}{\prod \xi_{j}} t^{i-n}
$$

Lemma 8. Let the circle act symplectically on a compact symplectic $2 n$-manifold $M$ with isolated fixed points. If the range of the Chern class map $\mathrm{c}_{1}(M)$ contains at most $n$ elements, then

$$
\sum_{\substack{p \in M^{S^{1}} \\ \mathrm{c}_{1}(M)(p)=k}} \frac{1}{\Lambda_{p}}=0 \quad \forall k \in \mathbb{Z} .
$$

Here, $\Lambda_{p}$ is the product of the weights (with multiplicity) in the isotropy representation $\mathrm{T}_{p} M$ for all $p \in$ $M^{S^{1}}$. 
Proof. Let

$$
\begin{gathered}
\left\{\mathrm{c}_{1}(M)(p) \mid p \in M^{S^{1}}\right\}=\left\{k_{1}, \ldots, k_{\ell}\right\} \subset \mathbb{Z}, \quad \text { and define } \\
A_{i}:=\sum_{\substack{p \in M^{S^{1}} \\
\mathrm{c}_{1}(M)(p)=k_{i}}} \frac{1}{\Lambda_{p}} \quad \forall i \in\{1, \ldots, \ell\} .
\end{gathered}
$$

Consider the $\ell \times \ell$ matrix $\mathcal{B}$ given by

$$
\mathcal{B}_{i j}:=\left(k_{i}\right)^{j-1} \quad \forall 1 \leq i \leq \ell \text { and } 1 \leq j \leq \ell .
$$

Since $\ell \leq n$ by assumption,

$$
\int_{M} \mathrm{c}_{1}(M)^{j}=0 \text { for all } j<\ell .
$$

Therefore, applying the ABBV localization formula (Theorem 7 ) to

$$
1, \mathrm{c}_{1}(M), \ldots, \mathrm{c}_{1}(M)^{\ell-1}
$$

gives a homogenous system of linear equations

$$
\mathcal{B} \cdot\left(A_{1}, \ldots, A_{\ell}\right)=(0, \ldots, 0) .
$$

Since $\mathcal{B}$ is a Vandermonde matrix, we have that

$$
\operatorname{det}(\mathcal{B}(\ell)) \neq 0 \text {. }
$$

Thus, it follows from (1) that

$$
A_{1}=\cdots=A_{\ell}=0
$$

Corollary 9. There does not exist a symplectic $S^{1}$-action with exactly one fixed point.

Proof of Theorem $\square$ If the Chern class map is somewhere injective, then there exists $k \in \mathbb{Z}$ so that

$$
\sum_{\substack{p \in M^{S^{1}} \\ \mathrm{c}_{1}(M)(p)=k}} \frac{1}{\Lambda_{p}} \neq 0 .
$$

By Lemma 8 , this implies that the range of the Chern class map contains at least $\frac{1}{2} \operatorname{dim} M+1$ elements; a fortiori, the action has at least $\frac{1}{2} \operatorname{dim} M+1$ fixed points.

Remark 10 More generally, let $s: M^{S^{1}} \rightarrow \mathbb{Z}$ be given by any product of Chern classes of total degree $2 \mathrm{~m}$. Then, by a nearly identical argument, if the range of $s$ contains at most $\frac{n}{m}$ elements then

$$
\sum_{\substack{p \in M^{S^{1}} \\ s(p)=k}} \frac{1}{\Lambda_{p}}=0 \quad \forall k \in \mathbb{Z} .
$$

In particular, $s$ is nowhere injective. 
Lemma 11. Let the circle act symplectically on a compact symplectic $2 n$-manifold with isolated fixed points. Then

$$
\left|\left\{p \in M^{S^{1}} \mid \lambda_{p}=i\right\}\right|=\left|\left\{p \in M^{S^{1}} \mid \lambda_{p}=n-i\right\}\right| \quad \forall i \in \mathbb{Z} .
$$

Here, $\lambda_{p}$ is the number of negative weights at $p$ for all $p \in M^{S^{1}}$.

Proof. By perturbing and rescaling the symplectic form if necessary, we may assume that there exists a circle valued moment map $\Phi: M \rightarrow S^{1}$ and that zero is a regular value [10, Lemma 1].

By cutting this manifold at 0 , we can construct a new symplectic orbifold $N$ with momentum map $\Psi: N \rightarrow \mathbb{R}$ with the property that the minimum and maximum fixed sets are each diffeomorphic to the reduced space

$$
M_{0}=\mu^{-1}(0) / S^{1} .
$$

On the complement of these extremal fixed point sets, $N$ is equivariantly symplectomorphic to $M \backslash \mu^{-1}(0)$.

Since $\Psi$ and $-\Psi$ are perfect Bott-Morse functions, we have equalities of Poincaré polynomials

$$
\begin{aligned}
& \mathrm{P}_{N}=\mathrm{P}_{M_{0}}+\sum_{p \in M^{S^{1}}} t^{\lambda_{p}}+t \mathrm{P}_{M_{0}} \quad \text { and } \\
& \mathrm{P}_{N}=\mathrm{P}_{M_{0}}+\sum_{p \in M^{S^{1}}} t^{n-\lambda_{p}}+t \mathrm{P}_{M_{0}} .
\end{aligned}
$$

The claim follows from (2) and (3).

By Poincaré duality, this has the following corollary

Corollary 12. Let the circle act symplectically on a compact symplectic $2 n$-manifold with $k$ isolated fixed points. If $k$ is odd, then $n$ is even.

We are now ready to prove our final result.

Lemma 13. Let the circle act symplectically on a compact symplectic manifold $M$ with isolated fixed points. Then

$$
\sum_{p \in M^{S^{1}}} \mathrm{~N}_{p}(\ell)=\sum_{p \in M^{S^{1}}} \mathrm{~N}_{p}(-\ell) \quad \forall \ell \in \mathbb{Z} .
$$

Here, $\mathrm{N}_{p}(\ell)$ is the multiplicity of $\ell$ in the isotropy representation $\mathrm{T}_{p} M$ for all weights $\ell \in \mathbb{Z}$ and all $p \in M^{S^{1}}$.

Proof. Assume that the lemma is true for all manifolds of dimension less than $\operatorname{dim} M$; we will prove that it holds for $M$.

Without loss of generality we may assume that $M$ is connected. By quotienting out by the subgroup which acts trivially, we may assume that the action is effective. Given a natural number $\ell \neq 1$, let $\mathbb{Z} /(\ell) \subset$ $S^{1} \subset \mathbb{C}$ be the natural subgroup generated by $e^{\frac{2 \pi i}{\ell}}$, and let $M^{\mathbb{Z} /(\ell)} \subset M$ denote the set of points which are fixed by $\mathbb{Z} /(\ell)$. Given any component $Z \subset M^{\mathbb{Z} /(\ell)}$ and any $p \in Z^{S^{1}}$, the multiplicity of $\ell$ (or $-\ell$ ) in the isotropy representation $\mathrm{T}_{p} Z$ is equal to the multiplicity of $\ell$ (or $-\ell$ ) in $\mathrm{T}_{p} M$. Since the action is effective, $\operatorname{dim} Z<\operatorname{dim} M$. Therefore, by the inductive hypothesis,

$$
\sum_{p \in Z^{S^{1}}} \mathrm{~N}_{p}(\ell)=\sum_{p \in Z^{S^{1}}} \mathrm{~N}_{p}(-\ell) \quad \forall \ell \in \mathbb{Z} \backslash\{-1,0,1\} .
$$


Since every fixed point lies in a unique component of $M^{\mathbb{Z} /(\ell)}$, it follows immediately that

$$
\sum_{p \in M^{S^{1}}} \mathrm{~N}_{p}(\ell)=\sum_{p \in M^{S^{1}}} \mathrm{~N}_{p}(-\ell) \quad \forall \ell \in \mathbb{Z} \backslash\{-1,0,1\} .
$$

Moreover, Lemma11 implies that

$$
\sum_{p \in M^{S^{1}}} \sum_{\ell=1}^{\infty} \mathrm{N}_{p}(-\ell)=\sum_{p \in M^{S^{1}}} \lambda_{p}=\sum_{p \in M^{S^{1}}}\left(\frac{1}{2} \operatorname{dim} M-\lambda_{p}\right)=\sum_{p \in M^{S^{1}}} \sum_{\ell=1}^{\infty} \mathrm{N}_{p}(\ell) .
$$

Here, $\lambda_{p}$ is the number of negative weights at $p$ for all $p \in M^{S^{1}}$. The result follows immediately.

Proof of Theorem 2 As we explained in the beginning of this section,

$$
\sum_{p \in M^{S^{1}}} \mathrm{c}_{1}(M)(p)=\sum_{\ell \in \mathbb{Z}} \sum_{p \in M^{S^{1}}} \ell \mathrm{N}_{p}(\ell)
$$

But Lemma 13 implies that the right hand side of this equation is equal to zero.

\section{Proof of Theorem 3}

In the proof, we will show that the set of weights in the isotropy representation $\mathrm{T}_{p} M$ contains a natural subset which satisfies the assumptions of the following technical lemma. It is easy to check that every such set contains at least three non-zero integers. With a little more work, we can describe these sets precisely.

Lemma 14. Let $\Sigma$ be a multiset of non-zero integers. Let $\mathrm{N}(\ell)$ denote the multiplicity of $\ell$ in $\Sigma$ for all $\ell \in \mathbb{Z}$. Assume that the following hold.

- $\mathrm{N}(\ell) \mathrm{N}(-\ell)=0$ for all $\ell \in \mathbb{Z}$.

- $\Sigma=-\Sigma \bmod \alpha$ for all $\alpha \in \Sigma$.

- $\sum_{\alpha \in \Sigma} \alpha=0$.

- There exists $n \in \mathbb{N}$ such that $\mathrm{N}(n)=1$ and $\mathrm{N}(\ell)=0$ for all $\ell \in \mathbb{Z}$ such that $|\ell|>n$.

Then there exist natural numbers $a$ and $b$ so that

$$
\Sigma=\{a+b,-a,-b\} .
$$

Proof. Let $k$ be the second largest number such that $\mathrm{N}(k)+\mathrm{N}(-k) \neq 0$. By assumption, there exist bijections $\theta: \Sigma \rightarrow \Sigma$ and $\theta^{\prime}: \Sigma \rightarrow \Sigma$ such that $\theta(\alpha)+\alpha=0 \bmod n$ and $\theta^{\prime}(\alpha)+\alpha=0 \bmod k$ for all $\alpha \in \Sigma$. Furthermore, we may assume that

$$
\theta^{2}=\left(\theta^{\prime}\right)^{2}=\operatorname{id}_{\Sigma}
$$

Define multisets

$$
\Sigma^{+}=\{\alpha \in \Sigma \mid \alpha>0\} \backslash\{n\} \quad \text { and } \quad \Sigma^{-}=\{\alpha \in \Sigma \mid \alpha<0\} .
$$


Let $\alpha \in \Sigma^{+}$. By assumption, $-\alpha \notin \Sigma$; in particular, $\theta(\alpha) \neq-\alpha$. Therefore, since $0<\alpha<n$ and $-n<\theta(\alpha) \leq n$, the fact that $\alpha+\theta(\alpha)=0 \bmod n$ implies that $\alpha+\theta(\alpha)=n$. In particular, $\theta(\alpha) \in \Sigma^{+}$. A nearly identical argument shows that, if $\alpha \in \Sigma^{-}$, then $\theta(\alpha) \in \Sigma^{-}$and $\alpha+\theta(\alpha)=-n$. Therefore,

$$
\sum_{\alpha \in \Sigma^{+}} \alpha=\frac{n}{2}\left|\Sigma^{+}\right| \quad \text { and } \quad \sum_{\alpha \in \Sigma^{-}} \alpha=-\frac{n}{2}\left|\Sigma^{-}\right|,
$$

where $\left|\Sigma^{ \pm}\right|$denotes the number of elements (counted with multiplicity) in $\Sigma^{ \pm}$. Finally, similar arguments apply to $\theta^{\prime}(\alpha)$ as long as $\theta^{\prime}(\alpha) \neq n$, or equivalently (since $\left(\theta^{\prime}\right)^{2}=\mathrm{id}_{\Sigma}$ ) as long as $\alpha \neq \theta^{\prime}(n)$. In particular, $\theta^{\prime}(\alpha) \in \Sigma^{+}$and $\alpha+\theta^{\prime}(\alpha)=k$ for all $\alpha \in \Sigma^{+} \backslash \theta^{\prime}(n)$, while $\theta^{\prime}(\alpha) \in \Sigma^{-}$and $\alpha+\theta^{\prime}(\alpha)=-k$ for all $\alpha \in \Sigma^{-} \backslash \theta^{\prime}(n)$. We now need to consider the two possible cases:

Case I: Assume $\mathrm{N}(k) \neq 0$.

In this case, by the paragraph above $k+\theta(k)=n$. Since $n+\theta^{\prime}(n)=0 \bmod k$, this implies that $\theta(k)+\theta^{\prime}(n)=0 \bmod k$. On the other hand, by assumption, $-\theta(k) \notin \Sigma$; in particular, $\theta^{\prime}(n) \neq-\theta(k)$. Since $0<\theta(k) \leq k$ and $-k<\theta^{\prime}(n) \leq n$ these two facts imply that $\theta^{\prime}(n)>0$. Therefore, by the preceding paragraph, $\theta^{\prime}(\alpha) \in \Sigma^{-}$and $\alpha+\theta^{\prime}(\alpha)=-k$ for all $\alpha \in \Sigma^{-}$. Hence,

$$
\sum_{\alpha \in \Sigma^{-}} \alpha=-\frac{k}{2}\left|\Sigma^{-}\right|
$$

Since $k \neq n$, this is a contradiction unless $\Sigma^{-}=\emptyset$. But this contradicts the assumption that $\sum_{\alpha \in \Sigma} \alpha=0$.

Case II: Assume $\mathrm{N}(-k) \neq 0$.

In this case, $-k+\theta(-k)=-n$, and so $\theta(-k)=-n \bmod k$. Therefore, we can choose $\theta^{\prime}$ so that $\theta^{\prime}(n)=\theta(-k)$. In particular, $\theta^{\prime}(n)<0$. By an argument analogous to that in the previous case, this is impossible unless $\Sigma^{+}=\emptyset$. Since $\alpha+\theta(\alpha)=-n$ for all $\alpha \in \Sigma^{-}$and $\sum_{\alpha \in \Sigma} \alpha=0$, this implies that there exist natural numbers $a$ and $b$ so that $\Sigma=\{a+b,-a,-b\}$.

We are now ready to show that Theorem 3 holds.

Proof of Theorem 3 If $\operatorname{dim} M=2$ then the quotient $M / S^{1}$ is a 1-dimensional manifold with boundary. Hence, $M / S^{1}$ is homeomorphic to the interval $[0,1] \subset \mathbb{R}$ and the fixed points correspond to $\{0\}$ and $\{1\}$. By Mayer-Vietoris, this implies that $H^{1}(M ; \mathbb{R})=0$, that is, $M$ is the 2 -sphere. Note that in this case, the ABBV localization formula implies that there exists a natural number $a$ so that the weights at the two fixed points are $a$ and $-a$.

Hence, we may assume that $\operatorname{dim} M>2$ and that the proposition is true for all manifolds of dimension less than $\operatorname{dim} M$; we will prove that it holds for $M$.

By quotienting out by the subgroup which acts trivially, we may assume that the action is effective. Let $p$ and $q$ be the fixed points. Let $\Sigma_{p}$ and $\Sigma_{q}$ denote the set of weights (counted with multiplicity) in the isotropy representations $\mathrm{T}_{p} M$ and $\mathrm{T}_{q} M$, respectively. Let $\mathrm{N}_{p}(\ell)$ and $\mathrm{N}_{q}(\ell)$ denote the multiplicity of $\ell$ in $\Sigma_{p}$ and $\Sigma_{q}$, respectively, for all $\ell \in \mathbb{Z}$. Finally, let $\Lambda_{p}$ and $\Lambda_{q}$ be the product of the weights in $\Sigma_{p}$ and $\Sigma_{q}$, respectively.

Let $n$ be the largest integer such that $\mathrm{N}_{p}(n)+\mathrm{N}_{q}(n) \neq 0$. By relabeling if necessary, we may assume that $\mathrm{N}_{p}(n) \neq 0$.

Claim I: $\mathrm{N}_{p}(\ell) \mathrm{N}_{p}(-\ell)=\mathrm{N}_{q}(\ell) \mathrm{N}_{q}(-\ell)=0$ for all $\ell \in \mathbb{Z} \backslash\{-1,0,1\}$. 
Since $|\ell|>1, \operatorname{dim} M^{\mathbb{Z} /(\ell)}<\operatorname{dim} M$. Moreover, the isotropy submanifold $M^{\mathbb{Z} /(\ell)}$ has at most two fixed points. Therefore, the inductive hypothesis and Corollary 9 together imply that $\ell$ and $-\ell$ can't both be weights in $\mathrm{T}_{p} M^{\mathbb{Z} /(\ell)}$ or in $\mathrm{T}_{q} M^{\mathbb{Z} /(\ell)}$; this proves the claim.

Claim II: $\Sigma_{p}=-\Sigma_{q}$.

By Claim $\mathrm{I}, \mathrm{N}_{p}(\ell) \mathrm{N}_{p}(-\ell)=\mathrm{N}_{q}(\ell) \mathrm{N}_{q}(-\ell)=0$ for all $\ell \in \mathbb{Z} \backslash\{-1,0,1\}$. By Lemma 13,

$$
\mathrm{N}_{p}(\ell)+\mathrm{N}_{q}(\ell)=\mathrm{N}_{p}(-\ell)+\mathrm{N}_{q}(-\ell) \quad \forall \ell \in \mathbb{Z} \backslash\{-1,0,1\} ;
$$

therefore $\mathrm{N}_{p}(\ell)=\mathrm{N}_{q}(-\ell)$ for all such $\ell$. The claim then follows immediately from Lemma 11, which implies that

$$
\sum_{\ell=1}^{\infty} \mathrm{N}_{p}(-\ell)=\lambda_{p}=\frac{1}{2} \operatorname{dim} M-\lambda_{q}=\sum_{\ell=1}^{\infty} \mathrm{N}_{q}(\ell) .
$$

Here, $\lambda_{p}$ and $\lambda_{q}$ are the number of negative weights in $\Sigma_{p}$ and $\Sigma_{q}$, respectively.

Claim III: $\Sigma_{p}=-\Sigma_{p} \bmod \alpha$ for all $\alpha \in \Sigma_{p}$.

Consider a nonzero integer $\ell$ and a component $Z \subset M^{\mathbb{Z} /(\ell)}$. By Corollary 9 if $Z$ is not a single point then it either contains both $p$ and $q$, or neither. In the former case, $\Sigma_{p}=\Sigma_{q} \bmod \ell$; see [15, Lemma 2.6]. Hence, $\Sigma_{p}=\Sigma_{q} \bmod \alpha$ for every $\alpha \in \Sigma_{p}$. Therefore, the claim follows immediately from Claim II.

Claim IV: $\sum_{\alpha \in \Sigma_{p}} \alpha=0$.

On the one hand, Lemma 8 implies that since $\operatorname{dim} M>2$,

$$
\mathrm{c}_{1}(M)(p)=\mathrm{c}_{1}(M)(q) \text { and } \quad \frac{1}{\Lambda_{p}}+\frac{1}{\Lambda_{q}}=0 .
$$

Moreover, by Theorem $2, \mathrm{c}_{1}(M)(p)+\mathrm{c}_{1}(M)(q)=0$; therefore

$$
\sum_{\alpha \in \Sigma_{p}} \alpha=\mathrm{c}_{1}(M)(p)=0
$$

Claim V: $n>1, \mathrm{~N}_{p}(n)=1$, and $\mathrm{N}_{p}(\ell)=0$ for all $\ell \in \mathbb{Z}$ such that $|\ell|>n$.

If the weights at $p$ and $q$ are all 1 or -1 , then the fact that $\mathrm{c}_{1}(M)(p)=\mathrm{c}_{1}(M)(q)$ implies that $\Lambda_{p}=\Lambda_{q}$. Since this contradicts (5), there exists $\ell \neq \pm 1$ so that $\mathrm{N}_{p}(\ell)+\mathrm{N}_{q}(\ell) \neq 0$. By Claim II and the definition of $n$, this implies that $n>1$ and $\mathrm{N}_{p}(\ell)=0$ for all $\ell \in \mathbb{Z}$ such that $|\ell|>n$.

Since $n>1, \operatorname{dim} M^{\mathbb{Z} /(n)}<\operatorname{dim} M$. Moreover, the isotropy submanifold $M^{\mathbb{Z} /(n)}$ has at most two fixed points. Therefore, the inductive hypothesis and Corollary 9 together imply that $\mathrm{N}_{p}(n)=1$.

Claim VI: $\Sigma_{p}=\{a+b,-a,-b\} \cup \bigcup_{i=1}^{m}\{1,-1\}$ for some natural numbers $a, b$, and $m$.

Let $\Sigma^{\prime}$ be the subset of $\Sigma_{p}$ formed by deleting the pair $\{1,-1\}$ as many times as possible, that is,

$$
\Sigma^{\prime}=\Sigma_{p} \backslash \cup_{i=1}^{m}\{1,-1\}, \quad \text { where } m=\min \left\{\mathrm{N}_{p}(1), \mathrm{N}_{p}(-1)\right\} .
$$

By Claims I, III, IV, and V, the set $\Sigma^{\prime}$ fulfills the requirements of Lemma 14, therefore, the claim follows immediately from that result. 
Claim VII: $\Sigma_{p}=\{a+b,-a,-b\}$ and $\Sigma_{q}=\{a, b,-a-b\}$ for some natural numbers $a$ and $b$.

By Claims II and VI,

$$
\Sigma_{p}=-\Sigma_{q}=\{a+b,-a,-b\} \cup \bigcup_{i=1}^{m}\{1,-1\}
$$

for some natural numbers $a, b$, and $m$. Therefore, since $\operatorname{dim}(M)=6+2 m$, to prove the claim is enough to we show that $\operatorname{dim}(M) \leq 6$. Moreover, by a straightforward computation,

$$
\left.c_{3}(M)\right|_{p}=-\left.c_{3}(M)\right|_{q}=(a+b) a b t^{3} \quad \text { and } \quad \Lambda_{p}=-\Lambda_{q}=(-1)^{m}(a+b) a b .
$$

Therefore, by the ABBV localization formula (Theorem 7),

$$
\int_{M} c_{3}(M)=\frac{(-1)^{m}}{t^{2 m}}+\frac{(-1)^{m}}{t^{2 m}}=\frac{2(-1)^{m}}{t^{2 m}} \neq 0 .
$$

As we explained in the beginning of Section 2 , this is impossible unless $\operatorname{dim} M \leq 6$, as required.

\section{References}

[1] M. Atiyah: Convexity and commuting Hamiltonians. Bull. London Math. Soc. 14 (1982) 1-15.

[2] M. Atiyah and R. Bott: The moment map and equivariant cohomology. Topology 23 (1984) 1-28.

[3] N. Berline and M. Vergne: Classes caractéristiques équivariantes. Formule de localisation en cohomologie équivariante. C. R. Acad. Sci. Paris 295 (1982) 539-541.

[4] T. Delzant: Hamiltoniens périodiques et images convexes de l'application moment. Bull. Soc. Math. France 116 (1988) 315-339.

[5] J.J. Duistermaat and A. Pelayo: Symplectic torus actions with coisotropic principal orbits. Ann. Inst. Fourier 57 (2007) 2239-2327.

[6] V. Ginzburg, V. Guillemin and Y. Karshon: Moment Maps, Cobordisms and Hamiltonian Group Actions. Appendix J by Maxim Braverman. Mathematical Surveys and Monographs, 98. American Mathematical Society, Providence, RI, 2002. viii+350 pp.

[7] L. Godinho: On certain symplectic circle actions. J. Symplectic Geom. 3 (2005) 357-383.

[8] V. Guillemin and S. Sternberg: Convexity properties of the moment mapping. Invent. Math. 67 (1982) 491-513.

[9] F. Kirwan: Convexity properties of the moment mapping. III. Invent. Math. 77 (1984) 547-552.

[10] D. McDuff: The moment map for circle actions on symplectic manifolds. J. Geom. Phys. 5 (1988) 149-160.

[11] D. McDuff and S. Tolman: Topological properties of Hamiltonian circle actions. IMRP Int. Math. Res. Pap. (2006) 72826, 1-77. 
[12] A. Pelayo: Symplectic actions of 2-tori on 4-manifolds. Mem. Amer. Math. Soc. 204 (2010) no. 959, viii+81p.

[13] A. Pelayo and S. Vũ Ngọc: Semitoric integrable systems on symplectic 4-manifolds. Inventiones Math. 177 (2009) 571-597.

[14] A. Pelayo and S. Vũ Ngọc: Constructing integrable systems of semitoric type. arXiv:0903.3376. Acta Mathematica, to appear.

[15] S. Tolman: On a symplectic generalization of Petrie's conjecture. To appear in Trans. Amer. Math. Soc. arXiv:0903.4918

[16] S. Tolman and J. Weitsman: On semi-free symplectic circle actions with isolated fixed points. Topology 39 (2000) 299-309.

[17] S. Vũ Ngọc: Moment polytopes for symplectic manifolds with monodromy. Adv. Math. 208 (2007) 909-934.

[18] J. Weitsman: A Duistermaat-Heckman theorem for symplectic circle actions. IMRN (1993) no. 12, 309-312.

\author{
Alvaro Pelayo \\ University of California-Berkeley \\ Mathematics Department, 970 Evans Hall \# 3840 \\ Berkeley, CA 94720-3840, USA. \\ E-mail: apelayo@math.berkeley.edu \\ Susan Tolman \\ UI Urbana-Champaign \\ Department of Mathematics \\ 1409 W Green St, Urbana (USA) \\ e-mail: stolman@math.uiuc.edu
}

